\title{
BMJ Open Casting versus flexible intramedullary nailing in displaced forearm shaft fractures in children aged 7-12 years: a study protocol for a randomised controlled trial
}

\author{
Petra Grahn (D) , ${ }^{1}$ Juha-Jaakko Sinikumpu, ${ }^{2}$ Yrjänä Nietosvaara, ${ }^{1,3}$ \\ Johanna Syvänen, ${ }^{4}$ Anne Salonen, ${ }^{5}$ Matti Ahonen, ${ }^{1}$ Ilkka Helenius ${ }^{6}$
}

To cite: Grahn P, Sinikumpu J-J, Nietosvaara Y, et al. Casting versus flexible intramedullary nailing in displaced forearm shaft fractures in children aged 7-12 years: a study protocol for a randomised controlled trial. BMJ Open 2021;11:e048248. doi:10.1136/ bmjopen-2020-048248

- Prepublication history for this paper is available online. To view these files, please visit the journal online (http://dx.doi. org/10.1136/bmjopen-2020048248).

Received 20 December 2020 Accepted 03 August 2021

Check for updates

(C) Author(s) (or their employer(s)) 2021. Re-use permitted under CC BY-NC. No commercial re-use. See rights and permissions. Published by BMJ.

For numbered affiliations see end of article.

Correspondence to

Dr Petra Grahn;

petra.grahn@hus.fi

\section{ABSTRACT}

Introduction The forearm is the most common fracture location in children, with an increasing incidence. Displaced forearm shaft fractures have traditionally been treated with closed reduction and cast immobilisation. Diaphyseal fractures in children have poor remodelling capacity. Malunion can cause permanent cosmetic and functional disability. Internal fixation with flexible intramedullary nails has gained increasing popularity, without evidence of a better outcome compared with closed reduction and cast immobilisation.

Method and analysis This is a multicentre, randomised superiority trial comparing closed reduction and cast immobilisation to flexible intramedullary nails in children aged $7-12$ years with $>10^{\circ}$ of angulation and/or $>10 \mathrm{~mm}$ of shortening in displaced both bone forearm shaft fractures (A0-paediatric classification: 22D/2.1-5.2). A total of 78 patients with minimum 2 years of expected growth left are randomised in 1:1 ratio to either treatment group. The study has a parallel non-randomised patient preference arm. Both treatments are performed under general anaesthesia. In the cast group a long arm cast is applied for 6 weeks. The flexible intramedullary nail group is immobilised in a collar and cuff sling for 4 weeks. Data are collected at baseline and at each follow-up until 1 year.

Primary outcome is (1) PROMIS paediatric upper extremity and (2) forearm pronation-supination range of motion at 1-year follow-up. Secondary outcomes are Quick DASH, Paediatric Pain Questionnaire, Cosmetic Visual Analogue Scale, wrist and elbow range of motion as well as any complications and costs of treatment.

We hypothesise that flexible intramedullary nailing results in a superior outcome.

Ethics and dissemination We have received ethical board approval (number: 78/1801/2020) and permissions to conduct the study from all five participating university hospitals. Informed consent is obtained from the parent(s). Results will be disseminated in peer-reviewed publications.

Trial registration number NCT04664517.
Strengths and limitations of this study

- First randomised controlled trial (RCT) to examine the treatment and outcome of displaced both bone forearm paediatric fractures.

- Multicentre RCT with blinded outcome assessors and recruiters.

- Use of several patient-reported outcome measures as well as active and passive upper limb range of motion.

- Bone age to determine remodelling capacity.

- Patient and treating surgeons not blinded.

\section{INTRODUCTION}

The incidence of paediatric forearm fractures is increasing, and almost half of all fractures in the growing skeleton are located in the forearm. ${ }^{1-3}$ Angular malunion exceeding $10^{\circ}$ can result in limited forearm rotation. ${ }^{4-6}$ The remodelling capacity of shaft fractures in children is poorer and less predictable than in metaphyseal and physeal fractures. More correction can be expected in children younger than 9years and in children with fractures close to the distal physes. ${ }^{78}$ The reported angular correction rate in the diaphysis is only $1^{\circ}-2^{\circ}$ per year until the end of growth, in malrotation probably even less. ${ }^{9-11}$

Active forearm rotation is restored during the first year after the fracture, after which regardless of remodelling very little change can be expected. ${ }^{12}$ Minimal total forearm rotation for performing activities of daily living in children and adolescents is considered to be $110^{\circ}\left(50^{\circ}\right.$ of supination, $65^{\circ}$ of pronation) and in adults $\left(50^{\circ}-60^{\circ}\right.$ of supination and $40^{\circ}-50^{\circ}$ of pronation).$^{13}{ }^{14}$ Loss of $\leq 60^{\circ}$ goes unnoticed in many patients. ${ }^{15}$ 


\begin{tabular}{lll}
\hline Table 1 & Pros and cons of the two treatment modalities \\
\hline & Pros & Cons \\
\hline Cast treatment & $\begin{array}{l}\text { Non-invasive } \\
\text { Cast wedging } \\
\text { Inexpensive }\end{array}$ & $\begin{array}{l}\text { Cast complications } \\
\text { Loss of reduction }\end{array}$ \\
& & $\begin{array}{l}\text { Frequent FU visits } \\
\text { Joint stiffness }\end{array}$ \\
& Loss of reduction rare & Invasive \\
& Low need for & Implant removal \\
& immobilisation & Infection \\
& Less FU visits & Risk for tendon/nerve \\
& Less affected ROM & injury \\
\hline
\end{tabular}

Modified from Mehlman and Wall. ${ }^{16}$

FIN, flexible intramedullary nail; FU, follow-up; ROM, range of motion.

Good forearm function has been documented in patients with $\leq 50^{\circ}$ of loss in pro-supination. ${ }^{79}$

Non-operative treatment (reduction and casting) is the golden standard in closed paediatric forearm shaft fractures, especially in children younger than 12 years of age. ${ }^{16}$ There is abundant evidence, that non-operative treatment of most forearm fractures gives satisfactory long-term cosmetic and functional outcomes. ${ }^{7}{ }^{16-19}$ Completely displaced both bone forearm fractures in older children and adolescents tend to be treated surgically, although evidence of successful outcome with cast treatment exists. ${ }^{20}$ Failure of reduction after closed treatment is higher in children $>10$ years of age and with fractures exceeding $15^{\circ}$ of angulation. ${ }^{22}{ }^{23}$

Flexible intramedullary nailing (FIN) has become the most popular method of internal fixation of paediatric forearm shaft fractures. FIN when applied correctly is minimally invasive surgery, with low complication rates compared with open reduction and internal fixation. ${ }^{20} 24$ Reported risk of iatrogenic complications varies between $9 \%$ and $31 \%^{725-30}$ with local wound infections and tendon injuries being the most common. Surgical management of paediatric forearm fractures has increased $>250 \%$ during the last two decades. ${ }^{31}{ }^{32}$ The two treatment modalities of displaced both bone forearm fractures have different benefits (table 1). Level I evidence regarding optimal treatment is missing. ${ }^{20}$ Different patient-reported outcome measures have been used, but only few are validated. ${ }^{33}$ The Patient-Reported Outcomes Measurement Information System (PROMIS) Paediatric Item Bank V.2.0 $0^{34}$-Upper Extremity is validated and has been used in prior paediatric upper extremity fracture studies. ${ }^{35}$

The aim of this study is to compare outcome of closed reduction and casting under general anaesthesia to internal fixation with FIN in paediatric displaced both bone forearm fractures. We hypothesise, that internal fixation of the fractures with FIN provides better functional outcome than closed reduction and cast treatment

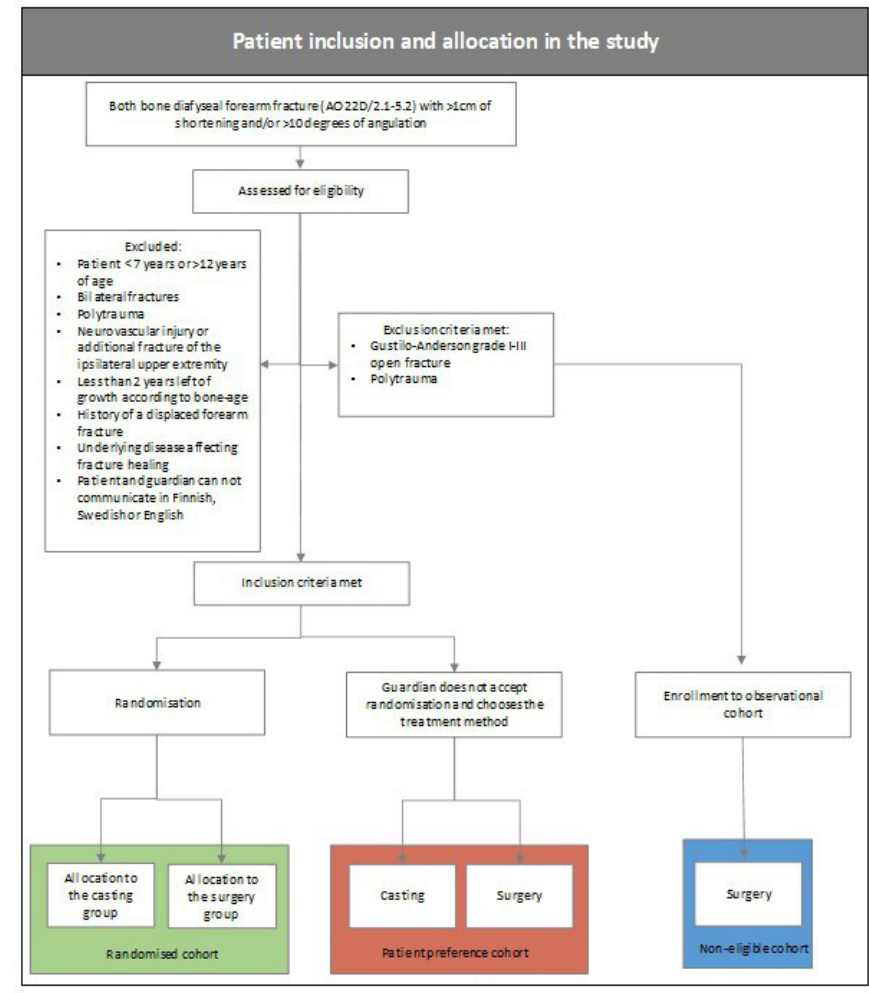

Figure 1 Eligibility screening, inclusion and exclusion criteria and patient allocation the study.

\section{METHODS AND ANALYSIS \\ Study design}

This is a multicentre parallel-group superiority randomised controlled trial (RCT) that complies with the Standard Protocol Items: Recommendations for Interventional Trials statement. The trial offers a patient choice arm (figure 1). The study is coordinated by Helsinki University Central Hospital, Children's Hospital unit for paediatric orthopaedics. Recruitment is done at all five Finnish university hospitals (Helsinki, Kuopio, Oulu, Tampere, Turku). The study is overseen by an external monitor provided by HUCH Clinical Research Institute (Clinical Research Institute HUCH, Helsinki, Finland, https://hyksinstituutti.fi/services/monitoring-services/? lang $=$ en).

Any changes in study protocol will be uploaded to the trial registry.

\section{Patient recruitment}

A specialist of either hand surgery, paediatric surgery, paediatric orthopaedics or orthopaedics screens all patients fitting the inclusion criteria for eligibility. If inclusion criteria are met, written consent is asked from the guardian. Patients and parents are given a written informed consent regarding the trial. The patient version is age adjusted for easier understanding according to the Finnish Investigators Network for Pediatric Medicines ( www.finpedmed.fi). 


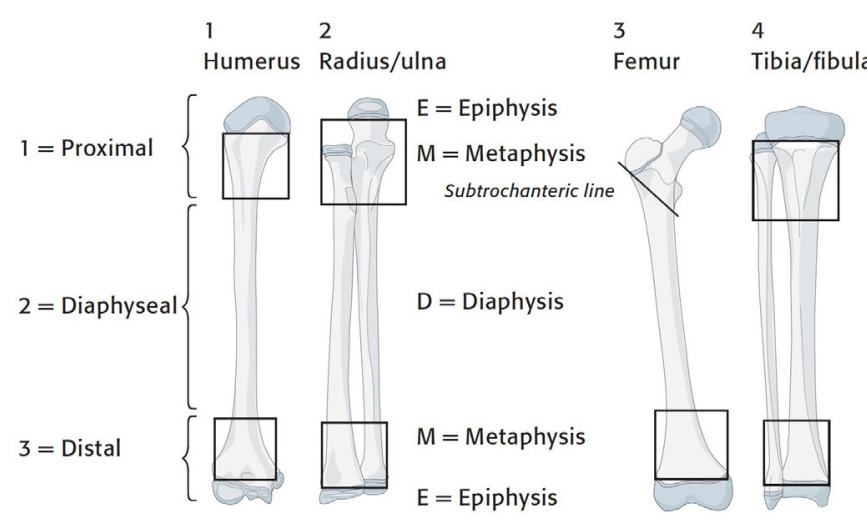

Figure 2 AO definition of the diaphyseal part of the forearm bones. Image copyright by AO Foundation, Switzerland. Figure reprinted with permission from the AO Foundation. Figure source: AO Surgery Reference (https:// surgeryreference.aofoundation.org).

\section{Eligibility criteria}

We will include all children aged 7-12years with open distal radial physis presenting with a both bone forearm shaft (diaphysis) fractures (AO-paediatric classification: $22 \mathrm{D} / 2.1-5.2)^{36}>10^{\circ}$ of angulation with or without $>10 \mathrm{~mm}$ of shortening (figures $1-3$ ). Patients with bilateral forearm fractures, Gustilo-Anderson grade I-III open fracture, ${ }^{37}$ ipsilateral upper limb fractures, neurovascular deficit, compartment syndrome, pathological fracture or patient not able to give a written informed consent will be excluded from the study (figure 1).

After agreeing to participation in the trial participants bone age is calculated from hand radiograph by the

\section{2-D Diaphyseal fractures}
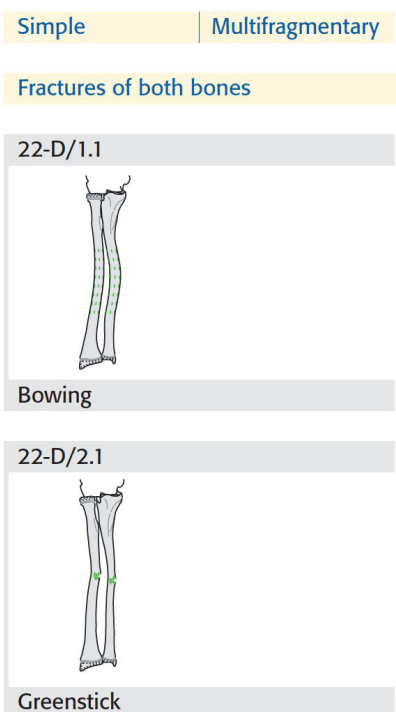

Figure $3 \mathrm{AO}$ forearm diaphysis fracture classification. Image copyright by AO Foundation, Switzerland. Figure reprinted with permission from the AO Foundation. Figure source: AO Surgery Reference (https://surgeryreference.aofoundation. org). method described by Greulich and Pyle ${ }^{38}$ by an experienced paediatric radiologist.

\section{Randomisation}

Included patients receive a temporary long arm cast supporting the fracture. Randomisation is done at treatment day 1 with patient under general anaesthesia in the operating theatre with the treating surgeon opening the assigned envelop. Prior to trial recruitment randomisation is performed at the main study for the expected trial population using a computer generated list. Assigned allocation (FIN or Cast) is sealed in individual coded envelopes. Randomisation ratio is 1:1, block size 10. Each trial centre is sent one block of envelopes at a time. Allocation sequence is kept at the main study centre and is not available to recruiting specialists.

\section{Patient's choice arm}

Patients who meet inclusion criteria, but refuse participation in the RCT are offered to choose the treatment method (reduction and casting or FIN). They continue through the trial in a prospective parallel patient preference arm, following the same treatment and follow-up (FU) protocol as the randomised patients (figure 4).

\section{Baseline}

Fracture displacement, angulation and patient bone age is calculated by an experienced paediatric radiologist unrelated to the trial from the lateral and anterioposterior (AP) radiographs of the forearm and AP radiograph of the hand. Date of injury, method of injury, patient's age at time of injury, sex, injured side, hand dominance and main sport or musical instrument as well as level are documented. Motor and sensory function of the injured side as well as range of motion (ROM) of uninjured arm (prosupination, elbow and wrist extension and flexion) are assessed.

\section{Intervention}

Time from injury to intervention (days), length of procedure (minutes) and surgeon's level of training (consultant, registrar) is documented.

\section{Cast group}

Fractures are reduced under general anaesthesia within 3 days from injury and a synthetic circular above elbow cast in neutral pro-supination is applied for 6 weeks. ${ }^{39}$ Final fracture alignment is documented with standard $\mathrm{AP}$ and lateral radiographs after cast application. If failure of reduction is observed at 10 days cast is wedged in the outpatient clinic followed by control radiographs (AP and lateral) of the forearm. Transfer to FIN group is recommended to all children, whose fracture alignment is not satisfactory after wedging (figure 4). Failure of reduction is defined as $>10^{\circ}$ of angulation on one or both bones in either AP and/or lateral radiograph, with or without $>10 \mathrm{~mm}$ of shortening as well as $>10 \mathrm{~mm}$ shortening in one or both bones alone. 


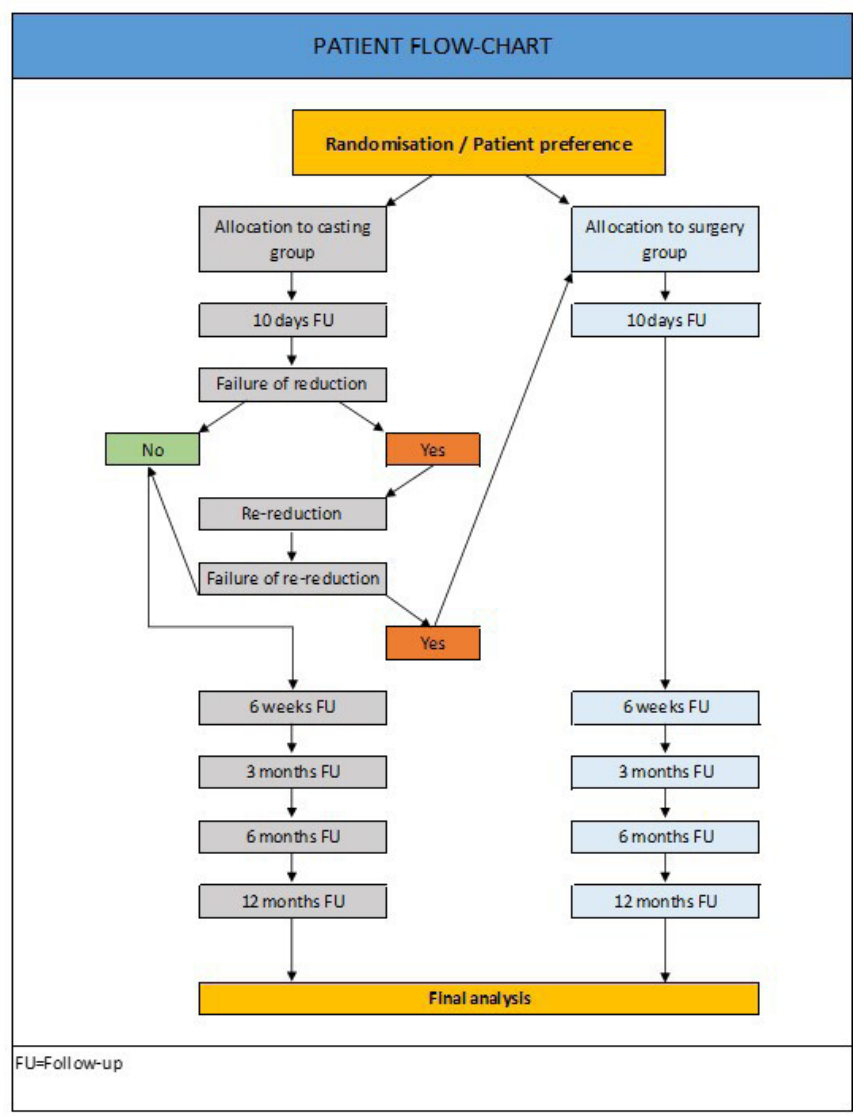

Figure 4 Patient flow chart in the study. Failure of reduction is defined as $>10^{\circ}$ of angulation on one or both bones in either AP and or lateral radiograph, with or without $>10 \mathrm{~mm}$ of shortening as well as $>10 \mathrm{~mm}$ shortening in one or both bones alone. If after wedging above criteria are met patients are transferred to flexible intramedullary nail group. AP, anterioposterior.

\section{FIN group}

Both fractures are reduced under general anaesthesia within 3 days of injury and a FIN is inserted to support the reduction. Nails size should be between $60 \%$ and $70 \%$ of the medullary canal of the radius or ulna measured in radiographs. Nails are inserted percutaneously, the radius nail radially, proximal to the physis, and the ulna nail from the lateral aspect of the olecranon, distal to the physis. If the nail cannot be passed across the fracture site within $15 \mathrm{~min}$ per fracture, open reduction is applied. Endcaps are not used, and nails are left under the skin. Final fracture alignment is documented with standard AP and lateral radiographs as well as nail placement distally and proximally with AP and lateral radiograph of the wrist and elbow. A collar and cuff sling is applied for 4 weeks. Sling can be removed daily, and upper limb actively moved through full ROM. Hardware is not routinely removed.

\section{Blinding}

This trial tests a clinical intervention that is not suitable for protection against treatment bias. Surgeon on duty at the emergency department will inform and recruit patients into the study. Recruiter will be blinded. The patient will be blinded to primary choice of treatment, but not to the treatment itself. Randomisation and allocation will be performed by the treating surgeon after induction of general anaesthesia. Trial data are collected at each appointment at the outpatient clinic by a physician not related to the trial, they will not be blinded. Main researchers will not participate in data collection. Statistician will be blinded.

\section{FU protocol and data collection}

Patients are examined at the paediatric orthopaedic outpatient clinic. Clinical and radiological $\mathrm{FU}$ for all patients regardless of treatment group is scheduled at 10 days, 6 weeks, 3 months, 6 months and 1 year (figure 3, table 2). Active and passive ROM of both forearms (forearm prosupination, wrist and elbow extension-flexion) is measured using a goniometer at all FU appointments for FIN group and from cast removal for cast group. Active and passive pro-supination is measured with the elbow was flexed at a $90^{\circ}$ angle with upper arm in adduction to eliminate compensation of potentially limited forearm pronation by shoulder abduction. The rotation measurements are taken from the distal forearm at the level of radius metaphysis to eliminate additional motion of the hand. ${ }^{40}$ Patients and guardians are requested to answer the following patient-reported outcome measures at each appointment; The PROMIS Paediatric Item Bank V.2.0Upper Extremity, ${ }^{34}$ QuickDASH,${ }^{41}$ Paediatric Quality of Life Inventory (PedsQL), ${ }^{42}$ PedsQL Paediatric Pain Questionnaire, ${ }^{22}$ Cosmetic Visual Analogue Scale (VAS 0-100).

Patients are allowed to commence full weight bearing when fracture union is observed (defined as three cortices out of four united). If fracture union is partial, but fractures are pain free on palpation, patients are allowed to commence non-weight-bearing activities with injured arm until next FU visit. If no signs of fracture unions are observed at 6 weeks (defined as no callus formation, painful on palpation) open reduction and internal plate fixation is recommended.

Time of returning to main sport or music and its level will be documented (weeks). Any adverse effects (wound infection, nerve or and tendon injury, delayed union, malunion and non-union) are documented as well as hardware problems and possible hardware removal as well as conversion of treatment during the $\mathrm{FU}$ period (cross-over from cast to FIN).

The data are collected using paper forms. The questionnaires will be completed at the outpatient clinic during the control visits. Research assistants will enter the data containing individual identification for each patient into two separate electronic databases located on a secure network drive. Data are protected with access codes known only by research assistants. Research assistants will contact patient and guardian if missing, implausible or inconsistent data are noticed.

\section{Outcome}

Primary outcome

1. PROMIS Paediatric Item Bank V.2.0-Upper Extremity at 1 year. ${ }^{34}$ 
Table 2 Diagram showing the data collection time points for all treatment groups; long arm cast and flexible intramedullary nail

\begin{tabular}{|c|c|c|c|c|c|c|c|}
\hline & \multicolumn{7}{|c|}{ Data collection time points } \\
\hline & Baseline & Treatment & $\begin{array}{l}\text { 1. Check-up } 10 \\
\text { days }\end{array}$ & $\begin{array}{l}\text { 2. Check-up } 6 \\
\text { weeks }\end{array}$ & $\begin{array}{l}\text { 3. Check-up } 3 \\
\text { months }\end{array}$ & $\begin{array}{l}\text { 4. Check-up } 6 \\
\text { months }\end{array}$ & $\begin{array}{l}\text { 5. Check-up } 12 \\
\text { months }\end{array}$ \\
\hline \multicolumn{8}{|l|}{ Long arm cast treatment } \\
\hline Diagnosis, eliqibility & $x$ & & & & & & \\
\hline Enrolment & $x$ & & & & & & \\
\hline Physical examination & $x$ & & & $\mathrm{x}$ & $x$ & $x$ & $x$ \\
\hline Bone age radiograph & & $x$ & & & & & \\
\hline $\begin{array}{l}\text { Standard radiograph (AP } \\
\text { and lateral) }\end{array}$ & $x$ & $\mathrm{x}^{*}$ & $x$ & $x$ & $x$ & $x$ & $x$ \\
\hline PedsQL pain & $x$ & & $x$ & $x$ & $x$ & $x$ & $\mathrm{x}$ \\
\hline PROMIS† & $x$ & & & $x$ & $x$ & $x$ & $x$ \\
\hline \multicolumn{8}{|l|}{$\begin{array}{l}\text { Flexible intramedullary nail } \\
\text { treamtment }\end{array}$} \\
\hline Diagnosis, eliqibility & $x$ & & & & & & \\
\hline Randomisation & $x$ & & & & & & \\
\hline Physical examination & $x$ & & & $x$ & $x$ & $x$ & $x$ \\
\hline Bone age radiograph & & $x$ & & & & & \\
\hline $\begin{array}{l}\text { Standard radiograph (AP } \\
\text { and lateral) }\end{array}$ & $x$ & $\mathrm{x}^{*}$ & & $x$ & $x$ & $x$ & $x$ \\
\hline PedsQL pain & $x$ & & $x$ & $x$ & $x$ & $\mathrm{x}$ & $x$ \\
\hline PROMIS & $x$ & & & $\mathrm{x}$ & $x$ & $\mathrm{x}$ & $x$ \\
\hline
\end{tabular}

*Standard lateral and AP radiograph taken after fracture fixation.

†PROMIS Paediatric Upper Extremity, PedsQL Main, QuickDASH.

AP, anterioposterior; PedsQL, Paediatric Quality of Life Inventory; PROM, patient-reported outcome measure.

2. Difference (\%) in forearm pronation-supination ROM at 1-year FU in comparison to uninjured side.

\section{Secondary outcome}

At all FU time points; Quick DASH, PROMIS Paediatric Item Bank V.2.0-Upper Extremity PedsQL, PEDS QL Pain module, Cosmetic VAS, difference in elbow and wrist extension/flexion and forearm pronation-supination in comparison to uninjured side. Malunion, delayed union (defined as nonunion at 3 months or later), pseudoarthrosis (defined as three cortices out of four not united at 6 months or later), need for reintervention other than wedging during 1-year FU, any adverse effects (wound infection, nerve and/or tendon damage). Level of practised sport or musical instrument before and at end of trial, as well as time from injury to return to hobby is registered.

\section{Statistical power calculation}

With two primary outcomes groups size was calculated for both, and then using the one requiring a larger cohort. We aim to collect 39 patients per group, taking into account a $10 \%$ drop-out rate.

\section{PROMIS Paediatric Item Bank V.2.0-Upper Extremity}

We aim to confirm that $75 \%$ of FIN patients have a better PROMIS UE score than what the mean score of the cast patients will be assuming both groups have the same SD and scores for both groups are normally distributed.
We will need at a minimum of 35 patients per group to achieve $80 \%$ power with significance level set at 0.05 for testing superiority of the FIN patients.

To account for potential drop-outs (10\%), we will collect an additional four patients per group. While this sample size calculation might not be optimal, due to assuming a normal distribution for the score, it is perhaps the only way we could estimate a required sample size in this case as we failed to find other analytical solutions for sample size estimation, and we did not have access to a similar data set to perform power simulations on.

\section{Difference (10\%) in forearm pronation-supination ROM}

For difference in prosupination, we aim for a $10 \%$ difference in the forearm prosupination in benefit for FIN group. Assuming SD $10 \%$ and $80 \%$ power the required amount of patients per group would be 16 .

\section{Statistical analyses}

We will perform all analyses using the intention-toprinciple. In case of significant cross-over a per-protocol analysis will be added. Randomisation will even the confounding factors, but if not, these will be adjusted using the multiple regression analyses. Our null hypothesis is that with FIN fixation superior outcome with is obtained.

After the final data set is formed from the primary data, data set access will be limited to the statistician and the 
authors of the final publication. The codes of the RCT arms will be known only to the research assistants until the blinded data interpretation has taken place.

If the study fails to collect the needed amount of patients as defined by the RCT protocol, patients from the 'patient preference arm' will be included in the final analysis, thus changing the status of the study from RCT to prospective.

\section{Patient and public involvement}

Patients, caregivers or public were neither involved in the development of the research questions nor the planning of the study design. They are neither involved in the recruitment nor conduct of the study. Results of the study are published only in peer-reviewed journals, no other information of the results of the study are provided to the patients or caregivers. Patients or caregivers will not take part in assessment regarding possible burden of the interventions of this study.

\section{Time schedule}

Patient enrolment started May 2021. We expect to have all patients enrolled by the end of the year 2022. The clinical part of the study will end with last FU visit of the last enrolled patient at the end of 2023.

\section{Ethics and dissemination}

We have obtained national Ethical committee approval (number: 78/1801/2020) for the study from the ethics committee of Southwest Finlands Hospital District (Hospital District of Southwest Finland Administrative Central, Kiinamyllynkatu 4-8, PO Box 52, FIN-20521 Turku, Finland). All five University hospitals (Helsinki, Kuopio, Tampere, Turku and Oulu) will apply for local institutional permission for taking part in this RCT. The study is run by Helsinki University Hospital, New Children's Hospital department of Pediatric Orthopedics and Traumatology. All patients and their guardians sign a written Informed consent before randomisation. Children with displaced forearm fractures will receive appropriate treatment. We will obtain all research data during the standard orthopaedic care of these children. Trail data will be maintained in secure storage at the research centre for 15 years after completion of study.

If at any point an imminent problem in healing is observed, warranting a change in the treatment regimen, this will be done at the discretion of the treating physician regardless of the initial treatment allocation. Trial will be ended at this time if following criteria will be met:

1. Conversion from either group will be over $25 \%$.

2. Serious adverse effects will be observed in more than $10 \%$ of each group.

The participants will be treated according to our best knowledge during and after the trial. Patients will not receive any compensation for participation. The Finnish Patient Insurance Centre will provide compensation for treatment injuries.
The findings of this study will be disseminated through peer-reviewed publications and conference presentations. Authorship will follow the International Committee of Medical Journal Editors (ICMJE) ${ }^{43}$ recommendations.

\section{Author affiliations}

${ }^{1}$ Department of Pediatric Orthopedics and Traumatology, Helsinki Children's Hospital, Helsinki University Hospital and University of Helsinki, Helsinki, Finland ${ }^{2}$ Department of Children and Adolescents, PEDEGO unit, University of Oulu and Oulu University Hospital, Oulu, Finland

${ }^{3}$ Department of Pediatric Surgery, Kuopio University Hospital, Kuopio, Pohjois-Savo, Finland

${ }^{4}$ Department of Pediatric Orthopedic Surgery, Turku University Hospital, Turku, Finland

${ }^{5}$ Department of Pediatric Surgery, Tampere University Hospital, Tampere, Finland ${ }^{6}$ Department of Orthopedics and Traumatology, University of Helsinki and HUS Helsinki University Hospital, Helsinki, Finland

Collaborators Arimatias Raitio, Turku University Hospital, Department of Pediatric Orthopedic Surgery (Turku, Finland) Jenni Jalkanen, Kuopio University Hospital, Department of Pediatric Surgery (Kuopio, Finland) Jussi Korhonen, Oulu University Hospital, Department of Children and Adolescents (Oulu, Finland).

Contributors PG, J-JS, YN, MA and IH have conceived and designed the study, performed the analysis and written the paper. JS and AS have participated in writing the paper.

Funding This research did not receive grants from any funding agency in the public, commercial or not-for-profit sectors. No direct or indirect funding will be acquired from industry related to this study. The authors have not declared a specific grant for this research from any funding agency in the public, commercial or not-for-profit sectors.

Competing interests $\mathrm{IH}$ reports grants from Medtronic and Stryker. $\mathrm{IH}$ is consulting surgeon at Medtronic. JS is consulting surgeon at Bioretec. None of the other authors report any conflict of interest.

Patient consent for publication Not required.

Provenance and peer review Not commissioned; externally peer reviewed.

Open access This is an open access article distributed in accordance with the Creative Commons Attribution Non Commercial (CC BY-NC 4.0) license, which permits others to distribute, remix, adapt, build upon this work non-commercially, and license their derivative works on different terms, provided the original work is properly cited, appropriate credit is given, any changes made indicated, and the use is non-commercial. See: http://creativecommons.org/licenses/by-nc/4.0/.

ORCID iD

Petra Grahn http://orcid.org/0000-0003-3018-8270

\section{REFERENCES}

1 Sinikumpu J-J, Serlo W. The shaft fractures of the radius and ulna in children: current concepts. J Pediatr Orthop B 2015;24:200-6.

2 Kapila R, Sharma R, Chugh A, et al. Evaluation of clinical outcomes of management of paediatric bone forearm fractures using titanium elastic nailing system: a prospective study of 50 cases. J Clin Diagn Res 2016;10:RC12-15.

3 Mäyränpää MK, Mäkitie O, Kallio PE. Decreasing incidence and changing pattern of childhood fractures: a population-based study. $J$ Bone Miner Res 2010;25:2752-9.

4 Tarr RR, Garfinkel Al, Sarmiento A. The effects of angular and rotational deformities of both bones of the forearm. An in vitro study. J Bone Joint Surg Am 1984;66:65-70.

5 Matthews LS, Kaufer H, Garver DF, et al. The effect on supinationpronation of angular malalignment of fractures of both bones of the forearm. J Bone Joint Surg Am 1982;64:14-17.

6 Thomas EM, Tuson KW, Browne PS. Fractures of the radius and ulna in children. Injury 1975;7:120-4.

7 Price CT, Scott DS, Kurzner ME, et al. Malunited forearm fractures in children. J Pediatr Orthop 1990;10:705-12.

8 Price CT. Acceptable alignment of forearm fractures in children: open reduction indications. J Pediatr Orthop 2010;30:S82-4. 
9 Fuller DJ, McCullough CJ. Malunited fractures of the forearm in children. J Bone Joint Surg Br 1982;64:364-7.

10 Daruwalla JS. A study of radioulnar movements following fractures of the forearm in children. Clin Orthop Relat Res 1979;139:114-20.

11 Hughston JC. Fractures of the forearm in children. J Bone \& Joint Surg Am 1962;44:1678-93.

12 Trousdale RT, Linscheid RL. Operative treatment of malunited fractures of the forearm. J Bone Joint Surg Am 1995;77:894-902.

13 Valone LC, Waites C, Tartarilla AB, et al. Functional elbow range of motion in children and adolescents. J Pediatr Orthop 2020;40:304-9.

14 Morrey BF, Askew LJ, Chao EY. A biomechanical study of normal functional elbow motion. J Bone Joint Surg Am 1981;63:872-7.

15 Högström H, Nilsson BE, Willner S. Correction with growth following diaphyseal forearm fracture. Acta Orthop Scand 1976;47:299-303.

16 Mehlman CT, Wall EJ. Diaphyseal radius and ulna fractures. In: Rockwood and Wilkins' Fractures in Children. Philadelphia: Wolters Kluwer Health, 2015: 413-72.

17 Sinikumpu J-J, Victorzon S, Antila E, et al. Nonoperatively treated forearm shaft fractures in children show good long-term recovery. Acta Orthop 2014;85:620-5.

18 Jones $\mathrm{K}$, Weiner DS. The management of forearm fractures in children: a plea for conservatism. J Pediatr Orthop 1999;19:811-5.

19 Blount WP. Forearm fractures in children. 1967. Clin Orthop Relat Res 2005;432:4-7.

20 Pace JL. Pediatric and adolescent forearm fractures. J Am Acad Orthop Surg 2016;24:780-8.

21 Zionts LE, Zalavras CG, Gerhardt MB. Closed treatment of displaced diaphyseal both-bone forearm fractures in older children and adolescents. J Pediatr Orthop 2005;25:507-12.

22 Bowman EN, Mehlman CT, Lindsell CJ, et al. Nonoperative treatment of both-bone forearm shaft fractures in children. $J$ Pediatr Orthop 2011;31:23-32.

23 Kay S, Smith C, Oppenheim WL. Both-bone midshaft forearm fractures in children. J Pediatr Orthop 1986:6:306-10.

24 Shah AS, Lesniak BP, Wolter TD, et al. Stabilization of adolescent both-bone forearm fractures: a comparison of intramedullary nailing versus open reduction and internal fixation. J Orthop Trauma 2010;24:440-7.

25 Kruppa C, Bunge P, Schildhauer TA, et al. Low complication rate of elastic stable intramedullary nailing (ESIN) of pediatric forearm fractures. Medicine 2017;96:e6669.

26 Kang S-N, Mangwani J, Ramachandran M, et al. Elastic intramedullary nailing of paediatric fractures of the forearm. J Bone Joint Surg Br 2011;93-B:262-5.

27 Fernandez FF, Langendörfer M, Wirth T, et al. Failures and complications in intramedullary nailing of children's forearm fractures. J Child Orthop 2010;4:159-67.
28 Smith VA, Goodman HJ, Strongwater A, et al. Treatment of pediatric both-bone forearm fractures. J Pediatr Orthop 2005;25:309-13.

29 Salonen A, Salonen H, Pajulo O. A critical analysis of postoperative complications of antebrachium ten-nailing in 35 children. Scand $J$ Surg 2012;101:216-21.

30 Antabak A, Luetic T, Ivo S, et al. Treatment outcomes of bothbone diaphyseal paediatric forearm fractures. Injury 2013;44 Suppl 3:S11-15

31 Helenius I, Lamberg TS, Kääriäinen S, et al. Operative treatment of fractures in children is increasing. J Bone Joint Surg Am 2009;91:2612-6.

32 Sinikumpu J-J, Lautamo A, Pokka T, et al. The increasing incidence of paediatric diaphyseal both-bone forearm fractures and their internal fixation during the last decade. Injury 2012;43:362-6.

33 Marson BA, Craxford S, Deshmukh SR, et al. Quality of patientreported outcomes used for quality of life, physical function, and functional capacity in trials of childhood fractures. Bone Joint $J$ 2020;102-B:1599-607.

34 Irwin DE, Gross HE, Stucky BD, et al. Development of six PROMIS pediatrics proxy-report item banks. Health Qual Life Outcomes 2012;10:10-22.

35 Gerull WD, Okoroafor UC, Guattery J, et al. Performance of pediatric PROMIS cats in children with upper extremity fractures. Hand 2020;15:194-200.

36 AO pediatric comprehensive classification of long bone fractures (PCCF). J Orthop Trauma 2018;32 Suppl 1:S117-40.

37 Gustilo RB, Anderson JT. Prevention of infection in the treatment of one thousand and twenty-five open fractures of long bones: retrospective and prospective analyses. J Bone Joint Surg Am 1976;58:453-8.

38 Radiographic atlas of skeletal development of the hand and wrist 1959

39 Noonan KJ, Price CT. Forearm and distal radius fractures in children. J Am Acad Orthop Surg 1998;6:146-56.

40 Korhonen L, Pokka T, Holappa A, et al. The measurement methods of movement and grip strength in children with a previous upper extremity fracture: a comparative, prospective research. Scand $J$ Surg 2020;109:351-8.

41 Beaton DE, Wright JG, Katz JN, et al. Development of the quickdash: comparison of three item-reduction approaches. J Bone Joint Surg Am 2005;87:1038-46.

42 Varni JW, Seid M, Rode CA. The PedsQL: measurement model for the pediatric quality of life inventory. Med Care 1999;37:126-39.

43 International Committee of Medical Journal Editors. Defining the role of authors and contributors. Available: http://www.icmje.org/ recommendations/browse/roles-and-responsibilities/defining-therole-of-authors-and-contributors.htm 